\title{
A NOTE ON CONVERGING GEOMETRIC-TYPE PROCESSES
}

\author{
MAXIM FINKELSTEIN, ${ }^{*}$ University of the Free State and \\ Max Planck Institute for Demographic Research
}

\begin{abstract}
The process of deterioration of repairable systems with each repair is modeled using converging geometric-type processes. It is proved that the expectation of the number of repairs in each interval of time is infinite. A new regularization procedure is suggested and the corresponding optimization problem is discussed.
\end{abstract}

Keywords: Geometric process; renewal process; repairable system; renewal function; minimal repair; overhaul

2010 Mathematics Subject Classification: Primary 60K10

Secondary $60 \mathrm{~N} 05$

\section{Introduction}

Deterioration in performance of imperfectly repaired items is often modeled using a stochastically decreasing sequence of lifetimes (cycles). This can be done in various ways. One popular approach is based on the virtual age concept, which states that after the repair action the age of a deteriorating item that started operation at $t=0$ and failed at $t=t_{f}$ is equal to some 'younger', intermediate value $t_{v}, 0 \leq t_{v} \leq t_{f}$. The case in which $t_{v}=0$ corresponds to the perfect repair, whereas $t_{v}=t_{f}$ means that a minimal repair has been performed. Different models exist for defining this intermediate value. The most popular method employs the linear reduction of the age at failure (see Kijima (1989), Doyen and Gadoin (2004), and Finkelstein (2007), to name a few). Note that the corresponding renewal-type process in this case is the process with dependent interarrival times that are governed by a generic cumulative distribution function (CDF) $F(t)$. On the other hand, a sequence of stochastically decreasing, independent lifetimes can also constitute a useful model for the deterioration of repairable items and the corresponding example will be considered in this paper.

Denote the duration of the $i$ th cycle by $X_{i} \geq 0, i=1,2, \ldots$, and the corresponding CDF by $F_{i}(t)$. Assume that the mean $\mu_{i}=\mathrm{E}\left[X_{i}\right]$ exists, and denote the variance by $\sigma_{i}^{2}$. Let $\mu_{i+1}<\mu_{i}$, which is obviously weaker than the (usual) stochastic ordering:

$$
X_{i+1}<_{\mathrm{st}} X_{i}, \quad i=1,2, \ldots,
$$

meaning that $F_{i}(t)>F_{i+1}(t), t>0$ (see Ross (1996)).

The geometric process introduced in Lam (1988) and thoroughly investigated in Lam (2007) is a meaningful example of (1). Let $Y_{1}, Y_{2}, \ldots$ be the independent and identically distributed sequence of continuous lifetime random variables with $\operatorname{CDF} F(t), F(0)=0$, mean $\mu$, and

Received 14 October 2009; revision received 15 March 2010.

* Postal address: Department of Mathematical Statistics, University of the Free State, 339 Bloemfontein 9300, South Africa. Email address: finkelm@ufs.ac.za 
variance $\sigma^{2}$. Then the geometric process is defined by the following sequence of random variables:

$$
X_{i}=\frac{Y_{i}}{a^{i-1}}, \quad i=1,2, \ldots,
$$

where $\mathrm{E}\left[X_{i}\right]=\mu / a^{i-1}, \operatorname{var}\left(X_{i}\right)=\sigma^{2} / a^{2(i-1)}$, and $a>0$ is a constant.

Note that relationship (2) is often equivalently written as $X_{i}=d^{i-1} Y_{i}, i=1,2, \ldots, d>0$, and called a 'quasi-renewal process', as in Wang and Pham (2006) and in their earlier papers, Wang and Pham (1996a), (1996b).

Let

$$
S_{n}=\sum_{i=1}^{n} X_{i}, \quad S_{0}=0 .
$$

Then the counting geometric process $N(t)$ is defined similarly to the ordinary renewal counting process, i.e.

$$
N(t)=\sup \left\{n: S_{n} \leq t\right\}, \quad t \geq 0 .
$$

When $a>1$,

$$
\sum_{i=1}^{n} \mathrm{E}\left[X_{i}\right]=\frac{\mu a}{a-1}<\infty,
$$

and the sequence $S_{n}, n \geq 1$, almost surely converges to

$$
S=\sum_{i=1}^{\infty} X_{i}, \quad S_{0}=0 .
$$

We will call this case the converging geometric process and we will discuss it (and its generalizations) in this paper.

As in ordinary renewal theory, the expectation $H(t) \equiv \mathrm{E}[N(t)]$ is of main interest. By analogy (see Lam (2007) and Wang and Pham (2006)), a similar general equation for the renewal function $H(t)$ holds:

$$
H(t) \equiv \mathrm{E}[N(t)]=\sum_{i=1}^{\infty} F^{(n)}(t),
$$

where $F^{(n)}(t)$ is the CDF of $S_{n}, n=1,2, \ldots$, and $F^{(1)}(t) \equiv F(t)$.

The concept of the converging geometric process can be generalized. Let

$$
X_{i}=\frac{Y_{i}}{q(i)}, \quad i=1,2, \ldots, a>1,
$$

where $q(i), q(1)=1$, is an increasing function of integer $i$ such that

$$
\sum_{i=1}^{\infty} \mathrm{E}\left[X_{i}\right]=b<\infty
$$

and, as previously, $Y_{1}, Y_{2}, \ldots$ are independent and identically distributed with governing CDF $F(t)$. Then, similar to (3) and (4), the corresponding geometric-type converging process 
can be defined. The specific case of (7) was considered in Braun et al. (2005), (2008) and called the series process:

$$
X_{i}=\frac{Y_{i}}{i^{a}}, \quad i=1,2, \ldots
$$

This sequence obviously converges (in the sense of (8)) for $a>1$.

In what follows, we will show that $H(t)=\infty$ for all $t>0$ and that this property 'does not prevent us' from using converging processes in reliability applications. In Section 3 we suggest a possible regularization and use it to obtain a simple optimal solution that can be of help when designing systems with two types of repair. This regularization, which has a clear practical meaning, results in a finite number of renewals in each interval of time. It is worth noting that the justification for using the geometric process as a tool for modeling sequences of lifetimes in practice was discussed in Section 4.1.3 of Wang and Pham (2006), whereas some aspects of statistical inference were investigated in Lam (1992).

\section{Infiniteness of $H(t)$ for converging processes}

The (renewal) function $H(t)$ defined in (6) plays a crucial role in ordinary renewal theory $(a=1)$. The following renewal-type integral equation was derived in Lam (1988) for the case of the geometric process (2):

$$
H(t)=F(t)+\int_{0}^{t} H(a(t-x)) \mathrm{d} F(x) .
$$

Using this equation, namely the specific form of the argument of the function $H(a(t-x))$, Braun et al. (2005) proved that, for $a>1$, the function $H(t)$ for all $t>0$ is infinite, which is not intuitively evident (intuitively, it is clear that this should be the case for sufficiently large $t$, e.g. for $t>\mu a /(a-1)$, as pointed out in Finkelstein (2008, pp. 76-79)). Note that (9) holds only for the geometric process. Therefore, the proof of the following theorem, which generalizes the result of Braun et al. (2005) to the geometric-type process (7)-(8), is based on a different concept.

Theorem 1. Assume that the governing lifetime CDF $F(t)$ is absolutely continuous, strictly positive, and strictly increasing for all $t>0$. Let the point process $N(t), t \geq 0$, be defined by the interarrival times described by (7) and (8). Then $\mathrm{E}[N(t)]$ is infinite for all $t>0$.

Proof. The first part of this proof is similar to that of Theorem 1 of Braun et al. (2005) for the geometric process (2).

(a) Denote the CDF of the random variable in (5) by $S(t)$. It follows from (8) that there exists $\varepsilon>0$ such that

$$
S(b) \equiv \operatorname{Pr}\left(\sum_{i=1}^{\infty} X_{i} \leq b\right)>\varepsilon .
$$

Indeed, if, on the contrary, $S(t)=0$ for $0 \leq t \leq b$, then

$$
\int_{0}^{\infty}(1-S(u)) \mathrm{d} u=\sum_{i=1}^{\infty} \mathrm{E}\left[X_{i}\right]>b,
$$

which contradicts (8). As

$$
\operatorname{Pr}\left(\sum_{i=1}^{n} X_{i} \leq b\right) \geq \operatorname{Pr}\left(\sum_{i=1}^{\infty} X_{i} \leq b\right)
$$


and

$$
N(b) \geq n \quad \Longleftrightarrow \quad \sum_{i=1}^{n} X_{i} \leq b,
$$

we have

$$
\operatorname{Pr}(N(b) \geq n)>\varepsilon, \quad n \geq 1,
$$

and, therefore,

$$
\mathrm{E}[N(b)]=\sum_{i=1}^{\infty} \operatorname{Pr}(N(b) \geq i)=\infty .
$$

As $H(t)$ is nondecreasing, it is also infinite for all $t \geq b$.

(b) Now we will prove that this property is valid for all $t>0$. Equation (5) can be rewritten as

$$
S=X_{1}+\sum_{i=2}^{\infty} X_{i}
$$

Denote the CDF of the sum on the right-hand side of this equation by $S_{2}(t)$, and consider the corresponding convolution:

$$
S(t)=\int_{0}^{t} f(x) S_{2}(t-x) \mathrm{d} x,
$$

where $f(t)=F^{\prime}(t)$. As

$$
\sum_{i=2}^{\infty} \mathrm{E}\left[X_{i}\right]=b-\mu,
$$

similar to (10), there exists $\varepsilon_{2}>0$ such that

$$
S_{2}(b-\mu) \equiv \operatorname{Pr}\left(\sum_{i=1}^{\infty} X_{i} \leq b-\mu\right)>\varepsilon_{2}>0,
$$

and, obviously, $S_{2}(t)>\varepsilon$ for $t>b-\mu$ as well. Our goal now is to reduce the value $t=b$ for which the function $H(t)$ was proved to be infinite. Let $t=b-\mu+\delta_{1}$, where $\delta_{1}$ is sufficiently small (its value will be discussed later). Then, taking into account the fact that $f(x)$ is strictly positive, (11) results in

$$
S\left(b-\mu+\delta_{1}\right)=\int_{0}^{b-\mu+\delta_{1}} f(x) S_{2}\left(b-\mu+\delta_{1}-x\right) \mathrm{d} x>\varepsilon_{2} \int_{0}^{\delta_{1}} f(x) \mathrm{d} x>0,
$$

which is written for the 'worst' scenario, when $S_{2}(t)=0$ for $0 \leq t<b-\mu$ and $S_{2}(t) \geq \varepsilon_{2}$ for $b-\mu \leq t \leq b-\mu+\delta_{1}$. Therefore, as previously,

$$
\mathrm{E}\left[N\left(b-\mu+\delta_{1}\right)\right]=\infty .
$$

Thus, at this step, we have decreased the 'point of infiniteness' from $b$ to $b-\mu+\delta_{1}$. We can proceed in a similar manner at the next step. Therefore,

$$
S=X_{1}+X_{2}+\sum_{i=3}^{\infty} X_{i}
$$


and

$$
S(t)=\int_{0}^{t} f^{(2)}(x) S_{3}(t-x) \mathrm{d} x
$$

where $f^{(2)}(t)=\mathrm{d} F^{(2)}(t) / \mathrm{d} t$ and $S_{3}(t)$ denotes the CDF of the sum in (14).

Taking into account (7) and (12),

$$
S_{2}\left(b-\mu-\frac{\mu}{q(2)}\right) \equiv \operatorname{Pr}\left(\sum_{i=1}^{\infty} X_{i} \leq b-\mu-\frac{\mu}{q(2)}\right)>\varepsilon_{3}>0 .
$$

Similarly to (13),

$$
\begin{aligned}
S\left(b-\mu-\frac{\mu}{q(2)}+\delta_{2}\right) & =\int_{0}^{b-\mu-\mu / q(2)+\delta_{2}} f^{(2)}(x) S_{2}\left(b-\mu-\frac{\mu}{q(2)}+\delta_{2}-x\right) \mathrm{d} x \\
& >\varepsilon_{3} \int_{0}^{\delta_{2}} f^{(2)}(x) \mathrm{d} x \\
& >0,
\end{aligned}
$$

where $\delta_{2}>0$. Therefore, as previously,

$$
\mathrm{E}\left[N\left(b-\mu-\frac{\mu}{q(2)}+\delta_{2}\right)\right]=\infty
$$

and we have decreased the 'point of infiniteness' to $b-\mu-\mu / q(2)+\delta_{2}$.

In the same manner this point can be decreased after the $n$th step to

$$
b-\mu-\left(\frac{\mu}{q(2)}+\cdots+\frac{\mu}{q(n)}\right)+\delta_{n} .
$$

Choosing the decreasing sequence of $\delta_{n}$ as

$$
\lim _{n \rightarrow \infty} \delta_{n}=0
$$

and taking into account the convergence in (8), we eventually arrive at the statement of the theorem.

\section{The process of imperfect repair}

In this section we consider only the converging geometric process. It will be shown that, although $H(t)=\infty$ for all $t>0$, this process after suitable regularization can be used to describe the sequences of deteriorating cycles in the process of imperfect repair. The regularization can be performed in several ways. Note that the repair action in practice is not instantaneous, which means that $H(t) \neq \infty$ automatically in this case. For instance, Lam (2007) considered another (increasing) geometric process for modeling the sequence of repair times, which makes sense in applications (see also, e.g. Stanley (1993) and Zhang (2002)). Another obvious method is based on the truncation of the geometric process when there cannot be more than $m \geq 1$ 'geometric renewals' in the process (see Wang and Pham (2006)). Our approach to regularization of the geometric process is different and it is based on the following reasoning. 
Assume that, with probability $\theta$, each (instantaneous) repair is perfect, i.e. the next cycle is distributed in accordance with the first cycle's CDF $F(t)$, and, with probability $1-\theta$, the repair results in the next cycle of the geometric process. The latter assumption means that if the current cycle is distributed in accordance with $F\left(a^{k-1} t\right), k=1,2, \ldots$, then the next cycle will have the CDF $F\left(a^{k} t\right)$. It is clear that the expected number of 'geometric repairs' between the perfect repairs is now finite, whereas the instances of perfect repairs constitute the corresponding ordinary renewal process. Note that this procedure somehow resembles the Brown-Proschan model of imperfect repair (see Brown and Proschan (1983) and Cha and Finkelstein (2009)).

Denote by $N_{\theta}$ the corresponding geometric random variable, which is equal to the number of geometric repairs between two consecutive perfect repairs plus 1 . Thus,

$$
\operatorname{Pr}\left(N_{\theta}=i\right)=\theta(1-\theta)^{i-1}, \quad i=1,2, \ldots
$$

As this is a geometric distribution, $\mathrm{E}\left[N_{\theta}\right]=1 / \theta$. The expectation of time between consecutive perfect repairs is

$$
\sum_{n=1}^{\infty}\left(\sum_{i=1}^{n} \frac{\mu}{a^{i-1}}\right) \operatorname{Pr}\left(N_{\theta}=n\right)=\mu \theta \sum_{n=1}^{\infty} \frac{1-c^{n}}{1-c}(1-\theta)^{n-1},
$$

where $c=1 / a$. When $\theta=1$ (only perfect repairs), this expectation is obviously equal to $\mu$ and we arrive at the ordinary renewal process with governing CDF $F(t)$. When $\theta$ is decreasing, (15) is increasing as more cycles appear between consecutive perfect repairs, whereas the limit (as $\theta \rightarrow 0$ ) is equal to $b$ defined in (8) (pure geometric process).

Now consider an optimal $\theta$, which minimizes the long-run operation costs of a system that is repaired in the described way. Let the cost of the imperfect repair be $C_{g}$, and let the cost of the overhaul (perfect repair) be $C_{p}\left(C_{g}<C_{p}\right)$.

Denote by $r_{c}(\theta)$ the long-run expected repair cost per unit of time (the repair cost rate). As usual in maintenance problems described by renewal-type processes, $r_{c}(\theta)$ is defined by a quotient. The numerator is the expected repair costs between consecutive perfect repairs (including, e.g. the first perfect repair and excluding the second perfect repair), whereas the denominator is just the expectation of the duration of this renewal cycle. Let, for notational convenience, $C_{g}=1$. Therefore,

$$
\begin{aligned}
r_{c}(\theta) & =\frac{(1 / \theta-1)+C_{P}}{\mu \theta \sum_{n=1}^{\infty}\left(1-c^{n}\right)(1-\theta)^{n-1} /(1-c)} \\
& =\frac{1 / \theta}{\mu \theta \sum_{n=1}^{\infty}\left(1-c^{n}\right)(1-\theta)^{n-1} /(1-c)}+\frac{\tilde{C}_{P}}{\mu \theta \sum_{n=1}^{\infty}\left(1-c^{n}\right)(1-\theta)^{n-1} /(1-c)},
\end{aligned}
$$

where $\tilde{C}_{P}=C_{P}-1$.

The function $r_{c}(\theta), \theta \in(0,1]$, can be easily qualitatively analyzed. The first quotient on the right-hand side of (16) decreases as $\theta$ increases. Indeed, the numerator is equal to the expectation of the number of geometric repairs (plus 1) between two consecutive perfect repairs. Therefore, it increases as $\theta$ decreases 'faster' than the denominator increases, because the mean of each subsequent cycle in the converging geometric process $(a>1)$ is smaller than the previous one. Alternatively, this can be easily shown by the corresponding differentiation. The second term increases as $\theta$ increases, because the denominator decreases in this case. 
The boundary values for the first term are as follows. When $\theta \rightarrow 0$, it tends to $\infty$, as the denominator tends to $b$ defined in (8); when $\theta=1$, it is equal to $1 / \mu$. For $\theta=0$, the second term is equal to $\tilde{C}_{P} / b$ and, for $\theta=1$, it is equal to $\tilde{C}_{P} / \mu$. Due to the 'structure' of the first term, $r_{c}(\theta)$ is decreasing, at least for sufficiently small $\theta$. On the other hand, for sufficiently large $\tilde{C}_{P}$, at least for values close or equal to $\theta=1$, the function $r_{c}(\theta)$ is increasing, as the derivative of the second term by increasing $\tilde{C}_{P}$ can be made as large as we wish. Obviously, when $\tilde{C}_{P}$ is sufficiently small, the first term in (16) 'prevails' and $r_{c}(\theta)$ is decreasing in $(0,1]$. The latter means that only overhauls $(\theta=1)$ should be performed in this case.

The function $r_{c}(\theta)$ is continuous in $(0,1]$. Therefore, if $\tilde{C}_{P}>C_{0}>0$, there exists $\theta_{m}$ such that

$$
r_{c}\left(\theta_{m}\right) \equiv \min _{\theta \in(0,1)} r_{c}(\theta)
$$

For the given values of parameters, the minimal value of $r_{c}(\theta)$ and the corresponding $\theta_{m}$ and $C_{0}$ can be found numerically.

The obtained result can be used for designing repairable devices with imperfect repair described by geometric repairs and overhauls. The value of the probability $\theta$ should be chosen in an optimal way in order to minimize the long-run costs. This can be the case, for instance, in systems where the proportion of failures that lead to overhauls (usually the most significant failures) can be controlled in the process of design. The cost of an overhaul as a result of these failures is also often large compared with the cost of the corresponding geometric repairs and, therefore, the condition $\tilde{C}_{P}>C_{0}$ usually holds in practice.

\section{Acknowledgement}

The author would like to thank an anonymous reviewer, whose suggestions helped to improve the presentation of this paper.

\section{References}

Braun, W. G., LI, W. And Zhao, Y. Q. (2005). Properties of the geometric and related processes. Naval Res. Logistics 52, 607-616.

Braun, W. G., LI, W. ANd Zhao, Y. Q. (2008). Some theoretical properties of the geo-metric and $\alpha$-series processes. Commun. Statist. Theory Meth. 37, 1483-1496.

Brown, M. and Proschan, F. (1983). Imperfect repair. J. Appl. Prob. 20, 851-859.

Cha, J. H. ANd Finkelstein, M. (2009). On a terminating shock process with independent wear Increments. J. Appl. Prob. 46, 353-362.

Doyen, L. AND Gaudoin, O. (2004). Classes of imperfect repair models based on reduction of failure intensity or virtual age. Reliab. Eng. System Safety 84, 45-56.

Finkelstein, M. (2007). On some ageing properties of general repair processes. J. Appl. Prob. 44, 506-513.

Finkelstein, M. (2008). Failure Rate Modelling for Reliability and Risk. Springer, London.

KiJima, M. (1989). Some results for repairable systems with general repair. J. Appl. Prob. 26, 89-102.

LAM, Y. (1988). Geometric process and the replacement problem. Acta Math. Appl. Sinica 4, 366-377.

LAM, Y. (1992). Nonparametric inference for geometric processes. Commun. Statist. Theory Meth. 21, $2083-2105$.

LAM,Y. (2007). The Geometric Process and Its Applications. World Scientific, Hackensack, NJ.

Ross, S. M. (1996). Stochastic Processes, 2nd edn. John Wiley, New York.

Stanley, A. D. J. (1993). On geometric processes and repair replacement problems. Microelectronic. Reliab. 33, 489-491.

Wang, H. And Pham, H. (1996a). Optimal maintenance policies for several imperfect repair models. Internat. J. Systems Sci. 27, 543-549.

Wang, H. AND Pham, H. (1996b). A quasi renewal process and its applications in imperfect maintenance. Internat. J. Systems Sci. 27, 1055-1062.

Wang, H. And Pham, H. (2006). Reliability and Optimal Maintenance. Springer, London.

ZHANG, Y. L. (2002). A geometric-process repair-model with good-as-new preventive repair. IEEE Trans. Reliab. 51, $223-228$. 